\title{
A New Relaxation Method for Binary Quadratic Programming: An Application to Densest k-subgraph
}

\author{
Chuanhao Guo ${ }^{1, *}$ and Liping Tang ${ }^{2}$ \\ ${ }^{1}$ School of Economics and Management, Zhejiang Sci-Tech University, Hangzhou 310018, China \\ ${ }^{2}$ College of Mathematics and Statistics, Chongqing Technology and Business University, Chongqing 400067, China \\ ${ }^{*}$ Corresponding author
}

\begin{abstract}
Binary quadratic programming (BQP) problem was an NP-hard problem and had a large number of applications. In this paper, a new relaxation method, that was doubly nonnegative relaxation, was proposed for solving BQP problem. Moreover, we prove that the doubly nonnegative relaxation for BQP is equivalent to a new tighter semidifinite relaxation. When BQP problem reduces to densest k-subgraph problem, the doubly nonnegative relaxation is equivalent to a tighter semidifinite relaxation. Finally, some comparative numerical results are reported to show that the efficiency of the doubly nonnegative relaxation is more promising than that of semidefinite relaxation for solving some specific BQP problems.
\end{abstract}

Keywords - binary quadratic programming; doubly nonnegative relaxation; semidefinite relaxation; densest $k$-subgraph

\section{INTRODUCTION}

In this paper, we consider the following binary quadratic programming (BQP) problem

$$
\begin{array}{lll} 
& \min & x^{T} Q x \\
(\mathrm{~B} \Theta \Pi) \quad \text { s.t. } & a_{i}^{T} x=b_{i}, \quad i=1,2, \mathrm{~K}, m, \\
& & x \in\{-1,1\}^{n},
\end{array}
$$

where $x \in R^{n}$ is the decision variable, $Q \in R^{n \times n}, c \in R^{n}, a_{i} \in R^{n}$ and $b_{i} \in R$ for all $i \in I:=\{1,2, \ldots, m\}$ are the data. Without loss of generality, $Q$ is symmetric, and we assume $Q$ is not positive semidefinite, which implies generally that problem (BQP) is nonconvex and NP-hard.

Problem (BQP) arises in many applications, such as financial analysis [1], molecular conformation problem [2] and cellular radio channel assignment [3]. Many combinatorial optimization problems are special cases of problem (BQP), such as max-cut (MC) problem [4], dense $k$-subgraph (DkS) problem [5]. For solving this type of problem, a systematic survey of the solution methods can be found in [6] of Chap. 10, and the references therein.

It is well-known that semidefinite relaxation is a powerful and computationally efficient approximation technique for solving a host of very difficult optimization problems, for instance, MC problem, boolean quadratic programming problem. It has also been at the center of some of the very exciting developments in the area of signal processing and communications [7]. The standard semidefinite relaxation for problem $(\mathrm{BQP})$ is

$$
\begin{aligned}
& \min X \bullet Q+2 c^{T} x \\
& \text { s.t. } \quad a_{i}^{T} x=b_{i}, \quad \forall i \in I \text {, } \\
& \text { (SDR) } \quad a_{i}{ }^{T} X a_{i}=b_{i}{ }^{2}, \quad \forall i \in I \text {, } \\
& X_{i i}=1, \quad \forall i=1,2, \mathrm{~K} n \text {, } \\
& X \in S_{n}^{+} \text {, }
\end{aligned}
$$

It is obviously that problem (SDR) is convex and gives a lower bound for problem (BQP) if the feasible set of problem (BQP) is nonempty. Moreover, if an optimal solution $\left(x^{*}, X^{*}\right)$ of problem (SDR) satisfies $X^{*}=x^{*}\left(x^{*}\right)^{T}$, then we can conclude that $x^{*}$ is also an exact optimal solution for problem (BQP).

Recently, Burer [11] presents a computable convex problem, i.e., doubly nonnegative programming which can be efficiently solved by some popular packages, to solving a large class of NP-hard nonconvex quadratic programs. For more details and developments of this technique, one may refer to [8$11]$ and the references therein.

Inspired by the idea in [11], the doubly nonnegative relaxation and the new semidefinite relaxation for problem (BQP) are established, respectively. We prove that the doubly nonnegative relaxation for problem (BQP) is equivalent to the new semidefinite relaxation. Applying this result to $\mathrm{DkS}$ problem, we obtain that the doubly nonnegative relaxation for DkS problem is equivalent to the new semidefinite relaxation. Moreover, some comparative numerical results are reported to illustrate the efficiency of doubly nonnegative relaxation and semidefinite relaxation, respectively.

\section{NEW RELAXATION FOR PROBLEM (BQP)}

For simplicity, let $A:=\left(a_{1}, a_{2}, \ldots, a_{\mathrm{m}}\right)^{\mathrm{T}}, b:=\left(b_{1}, b_{2}, \ldots, b_{m}\right), S_{n}$ denotes the cone of symmetric matrices, $S_{n}{ }^{+}$the cone of $n \times n$ positive semidefinite matrices, $N_{n}$ the cone of $n \times n$ nonnegative symmetric matrices and $e$ the vector of ones with appropriate dimension. $A \bullet B=\operatorname{trace}\left(A^{\mathrm{T}} B\right)$ for two conformal matrices $A$ and $B$. $\operatorname{diag}(M)$ is a column vector whose elements are the diagonal elements of the given matrix $M$. 


\section{A. Doubly Nonnegative Relaxation for Problem (BQP)}

Let $z=(e-x) / 2$ in problem (BQP), then $z \in\{0,1\}^{n}$, and problem (BQP) can be equivalently reformulated as

$$
\begin{array}{lll}
\min & 4 z^{T} Q z-4 z^{T}(Q e+c)+e^{T} Q e+2 c^{T} e \\
\text { (R-BQP) } & \text { s.t. } & 2 A z=A e-b, \\
& z \in\{0,1\}^{n} .
\end{array}
$$

According to Theorem 2.6 in [8], problem (R-BQP) can be further equivalently transformed into the following completely positive programming problem, where $C P_{1+n}$ is defined as follows

$$
C P_{1+n}:=\left\{X \in S_{1+n}: X=\sum_{k \in K} z^{k}\left(z^{k}\right)^{T}\right\}
$$

for some finite $\left\{z^{k}\right\}_{k \in K} \subset R_{1+n}^{+}$, where $C P_{1+n}$ is a closed convex cone, which is called completely positive cone. Thus problem (CPP) is a convex problem. However, problem (CPP) is NPhard, since checking whether or not a given matrix belongs to $C P_{1+n}$ is NP-hard [12]. Thus, it has to be replaced by some computable cones, which can efficiently approximate by $C P_{1+n}$.

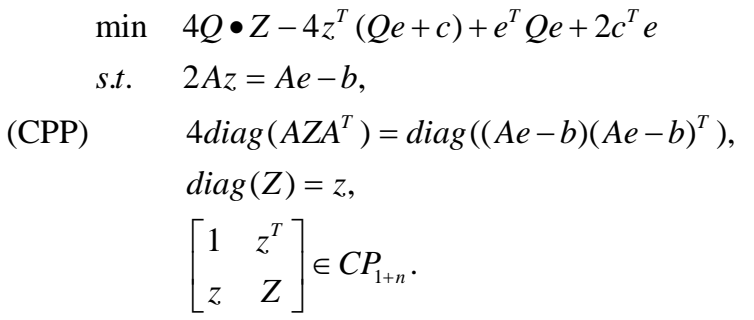

Note that the convex cone $N_{n}$ is self-dual, and so is the convex cone $S_{n}^{+}$. Hence, Diananda's decomposition theorem [13] can be reformulated as follows.

Theorem $1 C P_{n} \subseteq S_{n}^{+} \cap N_{n}$ holds for all $n$. If $n \leq 4$, then $C P_{n}=S_{n}^{+} \cap N_{n}$.

By the way, the matrices in $S_{n}^{+} \cap N_{n}$ sometimes are called doubly nonnegative. Of course, in dimension $n \geq 5$ there are matrices which are doubly nonnegative but not completely positive, the counterexample can be seen in [14]. By using Theorem 1, problem (CPP) can be relaxed to the following doubly nonnegative programming problem

$$
\begin{aligned}
\min & 4 Q \bullet Z-4 z^{T}(Q e+c)+e^{T} Q e+2 c^{T} e \\
\text { s.t. } \quad & 2 A z=A e-b, \\
(\mathrm{DNNP}) \quad & 4 \operatorname{diag}\left(A Z A^{T}\right)=\operatorname{diag}\left((A e-b)(A e-b)^{T}\right), \\
& \operatorname{diag}(Z)=z, \\
& {\left[\begin{array}{cc}
1 & z^{T} \\
Z & Z
\end{array}\right] \in S_{1+n}^{+} \cap N_{1+n}, }
\end{aligned}
$$

which is computable by some popular packages, such as CVX. Note that problem (DNNP) is similar to the semidefinite relaxation for problem (R-BQP), the only difference is exist in the last conic constraint. In the following subsection, problem (DNNP) will be compared with some semidefinite relaxation problems for problem (BQP) for solving some (BQP) instances.

\section{B. New Tighter Semidefinite Relaxation for Problem (BQP)}

Note that problem (BQP) can also be relaxed to the following problem

$$
\begin{array}{lll} 
& \min \quad & Q \bullet X+2 c^{T} x \\
& \text { s.t. } \quad & A x=b, \\
(\mathrm{R}-S D R) & & \operatorname{diag}\left(A X A^{T}\right)=\operatorname{diag}\left(b(b)^{T}\right), \\
& \operatorname{diag}(X)=e, \\
& X-x x^{T} \in S_{n}^{+} .
\end{array}
$$

If the optimal solution $\left(x^{*}, X^{*}\right)$ of problem (R-SDR) satisfies $X^{*}=x^{*}\left(x^{*}\right)^{T}$, it holds that $x^{*}$ is also an optimal solution of problem (BQP). On one hand, it is worth noting that

$$
X-x x^{T} \in S_{n}^{+} \Rightarrow X \in S_{n}^{+}
$$

always true, which implies that any feasible solution of problem (R-SDR) is also feasible for problem (SDR), i.e., Opt(SDR $) \leq$ Opt(R-SDR) since the two problems have the same objective functions, where $\operatorname{Opt}(\bullet)$ denotes optimal value for corresponding objective function. Therefore, we can conclude that problem (R-SDR) is the tighter semidefinite relaxation for problem (BQP) than problem (SDR).

On the other hand, we can easily verify that the constraint $X-x x^{T} \in S_{n}^{+}$is nonconvex, since the quadratic term $-x x^{T}$ is nonconvex. Thus, problem (R-SDR) is nonconvex and can not be solved by some popular packages for solving convex programs. In order to establish the convex representation for problem (R-SDR), a crucial theorem is given below and the details of its proof can be seen in Appendix A.5.5 Schur complement in [15].

Theorem 2 Let $M \in S_{n}$ is partitioned as $M=\left[\begin{array}{cc}D & B \\ B^{T} & C\end{array}\right]$. If $\operatorname{det}(D) \neq 0$, then the following relations hold, where $H=C-B^{T} D^{-1} B$, 
(i) $M \mathrm{f} O$ if and only if $\mathrm{Df} 0$ and $\mathrm{Hf} 0$;

(ii) If $D \mathrm{f} 0$, then $M \in S_{n}^{+}$if and only if $H \in S_{n}^{+}$.

By Theorem 2(ii) and (1), it holds immediately that

$$
\left[\begin{array}{cc}
1 & x^{T} \\
x & X
\end{array}\right] \in S_{1+n}^{+} \Leftrightarrow X-x x^{T} \in S_{n}^{+} \Rightarrow X \in S_{n}^{+} .
$$

So, problem (R-SDR) is equivalently reformulated as follows

$$
\begin{array}{ll}
\min & Q \bullet X+2 c^{T} x \\
\text { s.t. } & A x=b, \\
& \operatorname{diag}\left(A X A^{T}\right)=d \\
& \operatorname{diag}(X)=e, \\
& {\left[\begin{array}{cc}
1 & x^{T} \\
X & X
\end{array}\right] \in S_{1+n}^{+} .}
\end{array}
$$$$
\text { (SDR-1) } \quad \operatorname{diag}\left(A X A^{T}\right)=\operatorname{diag}\left(b(b)^{T}\right),
$$

which can be efficiently solved by some popular packages for solving convex programs. Here, some examples are tested to show that problem (SDR1) is a tighter relaxation for problem (BQP) compared with problem (SDR), and the corresponding numerical results also show that problem (SDR1) is more promising. These examples are solved by CVX [16], a package for specifying and solving convex programs.

Example 1 This is a two-dimensional nonconvex problem with one linear equality constraint, and the corresponding coefficients are given as follows

$$
Q=\left[\begin{array}{cc}
0 & -3 \\
-3 & -20
\end{array}\right], c=\left[\begin{array}{c}
-8 \\
9
\end{array}\right], A=[10,-10], b=0
$$

On one hand, by using CVX to solve problem (SDR), we obtain Opt $(\mathrm{SDR})=-\infty$, since problem $(\mathrm{SDR})$ is unbounded below. On the other hand, when problem (SDR1) is solved, one has Opt(SDR1)=-28 with $X=\left[\begin{array}{ll}1 & 1 \\ 1 & 1\end{array}\right]$ and $x=\left[\begin{array}{ll}-1, & -1\end{array}\right]^{T}$. Note that the relationship $X=x x^{T}$ holds, thus we can conclude that $x=[-1,-1]^{T}$ is also an optimal solution for problem (BQP). The results show that problem (SDR1) is more tight and efficient than problem (SDR) for solving this problem.

Example 2 The five-dimensional problem with three linear equality constraints, and the corresponding coefficients are chosen as follows

$$
\begin{gathered}
Q=\left[\begin{array}{ccccc}
-52 & 31 & 49 & -7 & 4 \\
31 & -16 & -50 & -13 & -49 \\
49 & -50 & 8 & 44 & -30 \\
-7 & -13 & 44 & 36 & 12 \\
4 & -49 & -30 & 12 & 56
\end{array}\right], c=\left[\begin{array}{c}
-20 \\
37 \\
43 \\
25 \\
-6
\end{array}\right] \\
A=\left[\begin{array}{ccccc}
4 & 10 & 29 & 14 & -36 \\
38 & 9 & 1 & -17 & 23 \\
48 & 39 & 5 & -17 & -13
\end{array}\right], b=\left[\begin{array}{c}
11 \\
-50 \\
-36
\end{array}\right] .
\end{gathered}
$$

If this problem is solved by problem (SDR), then it returns Opt(SDR) $=-\infty$ since problem (SDR) is unbounded below . When using problem (SDR1) to solve this problem, we have Opt(SDR1)=-307.548. However, the relationship $X=x x^{T}$ does not holds. Therefore, we obtain a tighter lower bound -307.548. These results also show that problem (SDR1) is more effective than problem (SDR).

In fact, the constraint $x \in\{-1,1\}^{n}$ in problem (BQP) implies

(SDR2)

$$
\begin{array}{ll}
\min & Q \bullet X+2 c^{T} x \\
\text { s.t. } \quad & A x=b, \\
& \operatorname{diag}\left(A X A^{T}\right)=\operatorname{diag}\left(b(b)^{T}\right), \\
& \operatorname{diag}(X)=e, \\
& 1-x_{i}-x_{j}+X_{i j} \geq 0, \quad \forall 1 \leq i \leq j \leq n, \\
& {\left[\begin{array}{cc}
1 & x^{T} \\
x & X
\end{array}\right] \in S_{1+n}^{+} .}
\end{array}
$$

that

$$
\left(1-x_{i}\right)\left(1-x_{j}\right) \geq 0 \Rightarrow 1-x_{i}-x_{j}+X_{i j} \geq 0
$$

for all $1 \leq i \leq j \leq n$. Substituting (3) into problem (SDR1), we can get the new semidefinite relaxation problem (SDR2).

Note that each feasible solution of problem (SDR2) is also feasible for problem (SDR1). Thus the lower bound of problem (SDR2) is more tight than that of problem (SDR1), since $n(n+1) / 2$ new inequality constraints are added into the corresponding problem (SDR2).

Now, we test some nonconvex instances to show that the lower bound of problem (SDR2) is tighter than that of problem (SDR1) from the computational point of view. Moreover, it is worth noting that problem (DNNP) has $n+n(n+1) / 2$ equality constraints more than problem (SDR1), and $n$ equality constraints more than problem (SDR2), respectively. Thus, the lower bound which obtained from problem (DNNP) is much greater than that one from problem (SDR1) and problem (SDR2), respectively. Two types of instances are tested. 
TABLE I. STATISTICS OF THE TEST INSTANCES

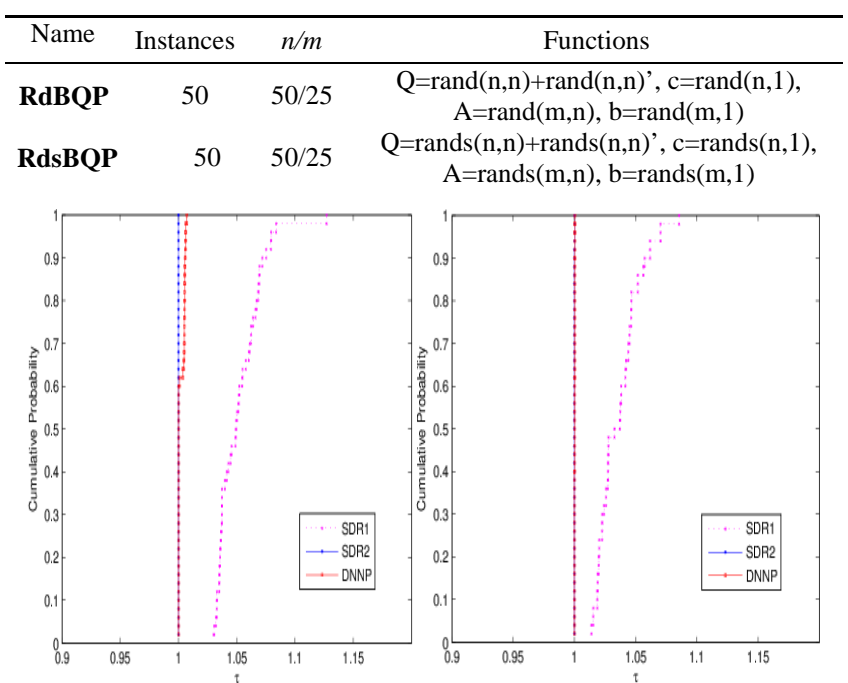

FIGURE I. THE PERFORMANCE OF LEFT FIGURE IS BASED ON OPTIMAL VALUES OF RDBQP, AND THE PERFORMANCE OF RIGHT FIGURE IS BASED ON OPTIMAL VALUES OF RDSBQP

To compare the performance of problems (SDR1), (SDR2) with that of problem (DNNP) for solving problem (BQP), we use the performance profiles described in Dolan and More's paper [17]. These instances are solved by CVX, and the results of performance are shown in FIGURE I. From FIGURE I, it is obviously that the performances of problem (SDR2) and problem (DNNP) are almost the same, which are better than that one of problem (SDR1), for solving RdBQP and RdsBQP, respectively. Thus, we can obtain that it is more efficient to use problem (SDR2) and problem (DNNP) for solving problem (BQP) than to use problem (SDR1), from the point of view of optimal values.

\section{The EQUiVALENCE BETWEen RELAXATION PROBLEMS}

In this section, we investigate the relationship between the two relaxation problems (DNNP) and (SDR2) First of all, the definition of the equivalence of two optimization problems is given as follows.

Definition 1 Two problems are called equivalent if they satisfy the following two conditions:

(i) If from a solution of one problem, a solution of the other problem is readily found, and vice versa. value.

(ii) The two problems have the same optimal objective

Now, based on the above Definition 1, the main theorem is given below.

Theorem 3. Suppose that the feasible sets Fea(DNNP) and Fea(SDR2) are both nonempty. Then two problems (DNNP)\} and (SDR2) are equivalent.

Proof: The proof can be divided into two parts. First of all, we will prove that $\mathrm{Opt}(\mathrm{SDR} 2) \geq \mathrm{Opt}(\mathrm{DNNP})$.

Suppose that $\left(x^{*}, X^{*}\right)$ is an optimal solution of problem (SDR2), let

$$
Z=\left(e e^{T}-e\left(x^{*}\right)^{T}-x^{*} e^{T}+X^{*}\right) / 4, z=\left(e-x^{*}\right) / 2 .
$$

By $A x^{*}=b$ and (4), we have

$$
A x^{*}=A(e-2 z)=b \Rightarrow 2 A z=A e-b .
$$

From (4) and $\operatorname{diag}\left(A X^{*} A\right)=\operatorname{diag}\left(b b^{T}\right)$, it follows that

$$
\begin{gathered}
\operatorname{diag}\left(A X^{*} A\right)=\operatorname{diag}\left(A\left(4 Z-e e^{T}+e\left(x^{*}\right)^{T}+x^{*} e^{T}\right) A^{T}\right) \text { i.e., } \\
=\operatorname{diag}\left(b b^{T}\right), \\
4 \operatorname{diag}\left(A Z A^{T}\right)=\operatorname{diag}\left((A e-b)(A e-b)^{T}\right) .
\end{gathered}
$$

Again from (4), one has

$$
\operatorname{diag}(Z)=\left(e-2 x^{*}+\operatorname{diag}\left(X^{*}\right)\right) / 4=z,
$$

since $\operatorname{diag}\left(X^{*}\right)=e$.

From $1-x_{i}^{*}-x_{j}^{*}+X_{i j}^{*} \geq 0, \forall 1 \leq i \leq j \leq n$, it holds that

$$
Z_{i j} \geq 0, \forall 1 \leq i \leq j \leq n,
$$

which combining with (7), it is true that

$$
z_{i} \geq 0, \forall 1 \leq i \leq n .
$$

By Theorem 2(ii) and (4), it follows that

$$
\begin{aligned}
Z-\mathrm{zZ}^{T}= & \left(e e^{T}-e\left(x^{*}\right)^{T}-x^{*} e^{T}+X^{*}\right) / 4 \\
& -\left(e-x^{*}\right)\left(e-x^{*}\right)^{T} / 4 \\
= & \left(X^{*}-x^{*}\left(x^{*}\right)^{T}\right) \in S_{n}^{+} .
\end{aligned}
$$

Combine (5), (6), (8), (9) with (10), it follows that $(z, Z)$ defined by (4) is a feasible solution for problem (DNP).

Moreover, again from (4), we have

$$
\begin{aligned}
& 4 Q \bullet Z-4 z^{T}(Q e+c)+e^{T} Q e+2 c^{T} e \\
& =Q \bullet\left(e e^{T}-e\left(x^{*}\right)^{T}-x^{*} e^{T}+X^{*}\right)-2\left(e-x^{*}\right)^{T}(Q e+c) \\
& \quad+e^{T} Q e+2 c^{T} e \\
& =Q \bullet X^{*}+2 c^{T} x^{*}=\operatorname{Opt}(\operatorname{SDR} 2),
\end{aligned}
$$

which further implies that Opt(DNNP) $\leq$ Opt(SDR2).

On the other hand, given an optimal solution $\left(z^{*}, Z^{*}\right)$ of problem (DNNP), and let 


$$
X_{i j}=1-2 z_{i}^{*}-2 z_{j}^{*}+4 Z_{i j}^{*}, x_{i}=1-2 z_{i}^{*},
$$

for all $1 \leq i \leq j \leq n$, which imply that

$$
\operatorname{diag}(X)=e-4 z^{*}+4 \operatorname{diag}\left(Z^{*}\right)=e,
$$

since $\operatorname{diag}\left(Z^{*}\right)=z^{*}$. Moreover, for $1 \leq i \leq j \leq n$,

$$
\begin{aligned}
1-x_{i}-x_{j}+X_{i j}= & 1-\left(1-2 z_{i}^{*}\right)-\left(1-2 z_{j}^{*}\right)+1-2 z_{i}^{*} \\
& -2 z_{j}^{*}+4 Z_{i j}^{*} \\
= & 4 Z_{i j}^{*} \geq 0 .
\end{aligned}
$$

From (11) and $2 A z^{*}=A e-b$, it follows that

$$
A x=A\left(e-2 z^{*}\right)=b .
$$

Again from (11), we have

$$
\begin{aligned}
\operatorname{diag}\left(A X A^{T}\right)= & \operatorname{diag}\left(A \left(e e^{T}-2 e\left(z^{*}\right)^{T}-2 z^{*} e^{T}\right.\right. \\
& \left.\left.+4 Z^{*}\right) A^{T}\right) \\
= & \operatorname{diag}\left(b b^{T}\right) .
\end{aligned}
$$

From (11) and Theorem 2.2(ii), it holds that

$$
\begin{aligned}
X-x x^{T}= & e e^{T}-2 z^{*} e^{T}-2 e\left(z^{*}\right)^{T}+4 Z^{*} \\
& -\left(e-2 z^{*}\right)\left(e-2 z^{*}\right)^{T} \\
= & 4\left(Z^{*}-z^{*}\left(z^{*}\right)^{T}\right) \in S_{n}^{+} .
\end{aligned}
$$

By (13), (14), (15) and (16), we can conclude that $(x, X)$ defined by (11) is a feasible solution for problem (SDR2). Furthermore, we have

$$
\begin{aligned}
X \bullet Q+2 c^{T} x= & \left(e e^{T}-2 e\left(z^{*}\right)^{T}-2 z^{*} e^{T}+4 Z^{*}\right) \bullet Q \\
& +2 c^{T}\left(e-2 z^{*}\right) \\
= & 4 Z^{*} \bullet Q-4\left(z^{*}\right)^{T}(Q e+c)+e^{T} Q e+2 c^{T} e \\
= & O p t(\mathrm{DNNP}),
\end{aligned}
$$

which implies that $\mathrm{Opt}(\mathrm{SDR} 2) \leq \mathrm{Opt}(\mathrm{DNNP})$.

Up to now, we prove that Opt(SDR2)=Opt(DNNP). By (4) and (11), it holds that optimal solutions of problem (SDR2) and problem (DNNP) can be representation each other. According to Definition 3.1, we can conclude that problem (DNNP) is equivalent to problem (SDR2).
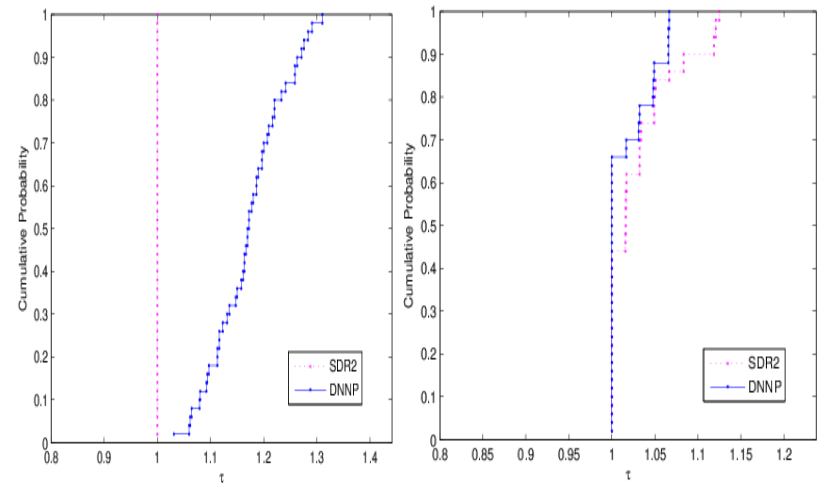

FIGURE II. THE PERFORMANCE OF LEFT FIGURE IS BASED ON THE NUMBER OF ITERATIONS OF RDBQP, AND RIGHT FIGURE IS BASED ON THE NUMBER OF ITERATIONS OF RDSBQP

Although Opt(SDR2)=Opt(DNNP) in view of Theorem 3 and Definition 1, problem (DNNP) has $n$ equality constraints more than problem (SDR2) in form. So, the amount of computation for solving problem (DNNP) may be much greater than that of for solving problem (SDR2). In order to illustrate this point of view, the compared performance results are shown in FIGURE II, which is based on the number of iterations for solving RdBQP and RdsBQP. The results in FIGURE II show that the performance of problem (SDR2) is better than that of problem (DNNP) for solving RdBQP, but the performance of problem (DNNP) is better than that of problem (SDR2) for solving RdsBQP, from the point of view of the number of iterations.

\section{An ApPlication: Densest K-SUBgraph PROBLEM}

The densest $k$-subgraph (DkS) problem [5] takes as input a graph $G$ on $n$ vertices and a parameter $k$, and asks for a subgraph of $G$ on at most $k$ vertices having the maximum total weight of edges. This problem is NP-hard and is a fundamental graph optimization problem and arises in several applications, such as community detection in social networks and identifying protein families [5], etc.

Given a symmetric $n \times n$ matrix $A=\left(a_{i j}\right)$. Let us associate a weighted graph with vertex set $\{1, \mathrm{~K}, n\}$ to $A$ in the usual way: If $i<j$ and $a_{i j} \neq 0$, we introduce the edge $[i, j]$ with weight $a_{i j}$ in the graph. Thus we interpret $A$ as the weighted adjacency matrix of a graph with vertex set $V=\{1, \mathrm{~K}, n\}$. The goal of DkS problem is to find a subset $V_{1}$ of $V$ consisting of $k$ vertices, such that the total weight of edges in the subgraph spanned by $V_{1}$ is maximized. This leads to DkS problem has the following formulation

$$
\begin{array}{lll} 
& \max & y^{T} A y \\
(\mathrm{DkS}) \quad \text { s.t. } & y^{T} e=k, \\
& y \in\{0,1\}^{n} .
\end{array}
$$

By using the techniques introduced in section 2, we can get doubly nonnegative relaxation and standard semidefinite relaxation problems for problem (DkS) as 


$$
\begin{aligned}
& \max A \bullet Y / 2 \\
& \text { s.t. } y^{T} e=k \text {, } \\
& \left(\mathrm{DNNP}_{\mathrm{DkS}}\right) \quad e^{T} Y e=k^{2} \text {, } \\
& \operatorname{diag}(Y)=y \text {, } \\
& {\left[\begin{array}{cc}
1 & y^{T} \\
y & Y
\end{array}\right] \in S_{1+n}^{+} \cap N_{1+n},}
\end{aligned}
$$

and

$$
\begin{aligned}
& \max A \bullet Y / 2 \\
& \text { s.t. } \quad y^{T} e=k \text {, } \\
& \left(\mathrm{I}_{-\mathrm{SDR}} \mathrm{DkS}\right) \quad e^{T} Y e=k^{2} \text {, } \\
& \operatorname{diag}(Y)=y \text {, } \\
& {\left[\begin{array}{cc}
1 & y^{T} \\
y & Y
\end{array}\right] \in S_{1+n}^{+},}
\end{aligned}
$$

respectively. Moreover, a new tighter semidefinite relaxation problem could be established for problem (DkS) as following

$$
\begin{aligned}
& \max \quad A \bullet Z / 8+Z^{T} A e / 4+e^{T} A e / 8 \\
\text { s.t. } \quad & Z^{T} e=2 k-n, \\
& e^{T} Z e=(2 k-n)^{2}, \\
& \operatorname{diag}(Z)=e, \\
& 1+Z_{i}+z_{j}+Z_{i j} \geq 0, \forall 1 \leq i \leq j \leq n, \\
& {\left[\begin{array}{cc}
1 & z^{T} \\
Z & Z
\end{array}\right] \in S_{1+n}^{+} . }
\end{aligned}
$$

\begin{tabular}{|c|c|c|}
\hline Name & & Functions \\
\hline A25 & $25 / 5$ & $\begin{array}{l}\text { seed }=1,2, \ldots, 50, \text { rand('seed', seed), A=round }\left(10^{*} \operatorname{rand}(n)\right), \\
\text { A25 }=\operatorname{tril}(A,-1)+\operatorname{triu}(A ', 0)\end{array}$ \\
\hline A30 & $30 / 10$ & $\begin{array}{l}\text { seed }=2012 \text {, randn('seed', seed), } A=\operatorname{randn}(n, n), A=\operatorname{tril}(A,- \\
1)+\operatorname{triu}(A \text { ' }, 0)\end{array}$ \\
\hline A40 & $40 / 15$ & $\begin{array}{l}\text { seed }=2017, \text { randn('seed', seed), } A=\operatorname{randn}(n, n), A=\operatorname{tril}(A, \\
-1)+\operatorname{triu}(A \text { ' }, 0)\end{array}$ \\
\hline A50 & $50 / 10$ & $\begin{array}{l}\text { seed }=2001, \quad \ldots, \quad 2050, \quad \text { randn('seed', seed), } \\
G=(\operatorname{rand}(n)<0.5), A=\text { round(20*rand(n)-10), A=tril(A, 1), } \\
A=A . * G, A 50=A+A \text { ' }\end{array}$ \\
\hline A60 & $60 / 20$ & $\begin{array}{l}\text { seed=2020, randn('seed', seed), A=round(randn(n,n)), } \\
A=\operatorname{tril}(A,-1)+\operatorname{triu}(A \text { ', 0) }\end{array}$ \\
\hline
\end{tabular}

Similar to the analysis of Theorem 3, we can obtain the following theorem immediately.

Theorem 4 Suppose that feasible sets Fea(DNNP $\left.{ }_{D k s}\right)$ and Fea(II-SDR $\left.{ }_{\text {DkS }}\right)$ are nonempty. Then the two problems $\left(\mathrm{DNNP}_{\mathrm{DkS}}\right)$ and $\left(\mathrm{III}_{-} \mathrm{SDR}_{\mathrm{DkS}}\right)$ are equivalent.

To give a flavour of the actual behaviour of the three relaxed problems for solving problem (DkS), some instances in TABLE II are tested.
TABLE II. STATISTICS OF THE TEST INSTANCES
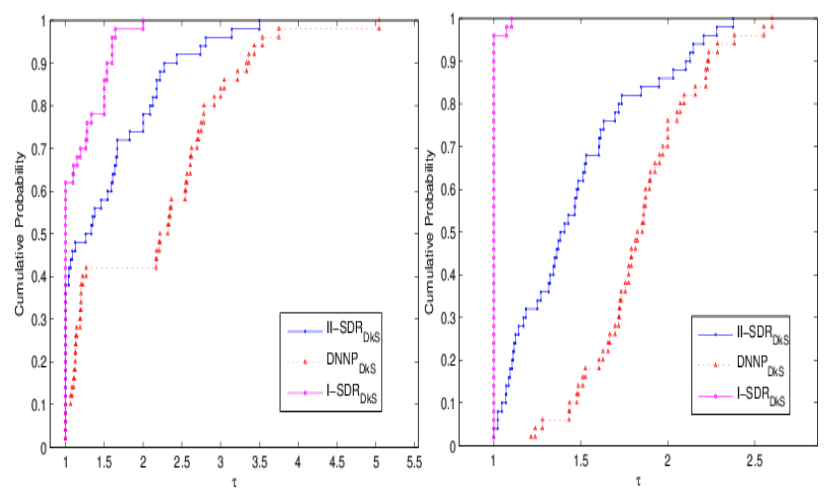

FIGURE III. THE PERFORMANCE PROFILE IS BASED ON THE NUMBER OF ITERATIONS OF PROBLEMS (DNNPDKS), (IISDRDKS) AND (I-SDRDKS), RESPECTIVELY, FOR SOLVING

$$
\text { A25 AND A50 }
$$

The performance results for solving A25 and A50 are shown in FIGURE III. In view of the number of iterations, the results in FIGURE III show that the performance of problem (I-

\begin{tabular}{|c|c|c|c|c|}
\hline & Model & Values & Number of iterations & CPU time \\
\hline \multirow[t]{3}{*}{ A30 } & $\left(\mathrm{DNNP}_{\mathrm{DkS}}\right)$ & 30.5894 & 52 & 3.4 \\
\hline & $\left(\mathrm{II}^{-S D R} \mathrm{DkS}_{\mathrm{DS}}\right)$ & 30.5903 & 70 & 5.0 \\
\hline & $\left(\mathrm{I}^{-S D R} \mathrm{RkS}_{\mathrm{D}}\right)$ & 33.3382 & 33 & 0.7 \\
\hline \multirow[t]{3}{*}{ A40 } & $\left(\mathrm{DNNP}_{\mathrm{Dks}}\right)$ & 74.4657 & 50 & 14.7 \\
\hline & (II-SDR $\left.{ }_{\mathrm{DkS}}\right)$ & 74.4658 & 66 & 20.6 \\
\hline & $\left(\mathrm{I}^{-S D R} \mathrm{R}_{\mathrm{DkS}}\right)$ & 75.5198 & 33 & 1.5 \\
\hline \multirow[t]{3}{*}{ A60 } & $\left(\mathrm{DNNP}_{\mathrm{DkS}}\right)$ & 150.385 & 52 & 105.5 \\
\hline & $\left(\right.$ II-SDR $\left.{ }_{\text {Dks }}\right)$ & 150.387 & 60 & 125.3 \\
\hline & $\left(\mathrm{I}^{-S D R} \mathrm{DkS}_{\mathrm{DS}}\right)$ & 154.272 & 40 & 3.2 \\
\hline
\end{tabular}
$\left.\mathrm{SDR}_{\mathrm{DkS}}\right)$ is better than that of problems $\left(\mathrm{DNNP}_{\mathrm{DkS}}\right)$ and (II$\mathrm{SDR}_{\mathrm{DkS}}$ ) for solving A25 and A50. According to Theorem 4, problems $\left(\mathrm{DNNP}_{\mathrm{DkS}}\right)$ and (II-SDR $\left.\mathrm{Dks}\right)$ can give more tight bounds for A25 and A50, while the number of iterations is a bit more. Note that the performance of problem (II-SDR ${ }_{D k S}$ ) is better than that of problem $\left(\mathrm{DNNP}_{\mathrm{DkS}}\right)$ in the viewpoint of the number of iterations, for A25 and A50.

In TABLE III, we report the corresponding comparative numerical results of three test instances from the viewpoint of optimal values and the number of iterations, respectively. The results show that the efficiency of problem $\left(\mathrm{DNNP}_{\mathrm{DkS}}\right)$ is better than that of problem (II-SDR $R_{\mathrm{DkS}}$ ). Thus we can conclude that it may be more promising to use problem (DNNP $\left.{ }_{\mathrm{Dks}}\right)$ than to use problem (II-SDR ${ }_{\mathrm{DkS}}$ ) for solving some specific (DkS) problems in practice.

TABLE III. NUMERICAL RESULTS OF INSTANCES 


\section{CONCLUDING REMARKS}

In this paper, a class of problems (BQP) is considered, which is NP-hard in general. In order to solve this class of problems, two types of convex relaxation methods are proposed. One of the methods is semidefinite relaxation. The other method is doubly nonnegative relaxation. Moreover, the two convex relaxation problems are equivalent. Applying these results to problem (DkS), we have the doubly nonnegative relaxation for problem (DkS) is equivalent to a new tighter semidefinite relaxation. Furthermore, the reported comparative numerical results show that the efficiency of doubly nonnegative relaxation is more promising than that of semidefinite relaxation for solving some specific (BQP) problems.

\section{ACKNOWLEDGMENT}

This work is supported by National Natural Science Foundation of China (Grant No. 11501350) and Science Foundation of Zhejiang Sci-Tech University (ZSTU) (Grant No. 16092098-Y).

\section{REFERENCES}

[1] R. D. McBride, J. S. Yormark, “An implicit enumeration algorithm for quadratic integer programming”, Manage. Sci., pp.282-296, 1980.

[2] A. T. Phillips, J. B. Rosen, "A quadratic assignment formulation of the molecular conformation problem”, J. Global Optim., vol. 4, pp. 229-241, 1994.

[3] P. Chardaire, A. Sutter, "A decomposition method for quadratic zero-one programming”, Manage. Sci.. pp. 704-712, 1995.

[4] M. X. Goemans, D. P. Williamson, "Improved approximation algorithms for maximum cut and satisfiability problems using semidefinite programming”, J. ACM., vol. 42, pp. 1115-1145, 1995.

[5] U. Feige, G. Kortsarz, D. Peleg, "The Dense k-Subgraph Problem Algorithmica”, vol. 29, pp. 410-421, 2001.

[6] D. Li, X. L. Sun, “Nonlinear Integer Programming”, Springer, New York 2006.

[7] W. K. Ma, C. C. Su, J. Jalden, T. H. Chang, C. Y. Chi, “The equivalence of semidefinite relaxation MIMO detectors for higher-order QAM", IEEE J-STSP,vol. 3, pp. 1038-1052, 2009.

[8] S. Burer, "On the copositive representation of binary and continuous nonconvex quadratic Programs”, Math. Program., vol. 120, pp. 479-495, 20009.

[9] N. Arima, S. Kim, M. Kojima, "Extension of completely positive cone relaxation to moment cone relaxation for polynomial optimization”, J. Optim. Theory App., vol. 168, pp. 884-900, 2016.

[10] Y. Q. Bai, C. H. Guo, "Doubly nonnegative relaxation method for solving multiple objective quadratic programming problems”, J. Ind. Manag. Optim., vol. 10, pp. 543-556, 2014.

[11] S. Burer, "Optimizing a polyhedral-semidefinite relaxation of completely positive programs”, Math. Program Comput., vol. 2, pp. 1-19, 2010.

[12] P. J. C. Dickinson, L. Gijben, "On the computational complexity of membership problems for the completely positive cone and its dual”, Comput. Optim. Appl., vol. 57, pp. 403-415, 2014.

[13] P. H. Diananda, "On non-negative forms in real variables some or all of which are non-negative”, Proc. Cambridge Philos. Soc. 58: 17-25, 1962.

[14] A. Berman, N. Shaked-Monderer, "Completely Positive Matrices”, World Scientific Pub. Co. Inc, 2003.

[15] S. P. Boyd, L. Vandenberghe, “Convex Optimization”, Cambridge University Press, 2004.

[16] M. Grant, S. Boyd, CVX: Matlab Software for Disciplined Convex Programming, version 1.21 http://cvxr.com/cvx, 2011.
[17] E. D. Dolan, J. J. More, "Benchmarking optimization software with performance profiles”, Math. Program. Vol. 91, pp. 201-213, 2002. 